\title{
Quantum dynamics of the sine-Gordon model in the presence of dissipation
}

\author{
Kazuo Hida* and Ulrich Eckern \\ Institut für Theorie der Kondensierten Materie, Universität Karlsruhe, \\ D-7500 Karlsruhe, Federal Republic of Germany
} (Received 9 July 1984)

\begin{abstract}
The influence of dissipation on the quantum soliton-antisoliton pair creation rate $P$ is calculated for the sine-Gordon model using the path-integral formulation. The external field dependence of $P$ is shown to change drastically in the weak-field limit. The relation to the electric conductivity of commensurability pinned charge-density waves is discussed.
\end{abstract}

Tunneling phenomena in nonlinear quantum systems have been attracting the attention of many authors as a typical example of essentially quantum phenomena. In systems with infinite degrees of freedom, tunneling has been discussed in terms of the "fate of the false vacuum." On the other hand, $\mathrm{Maki}^{2}$ suggested that the electric conduction in commensurate charge-density wave (CDW) systems is related to the creation of soliton-antisoliton pairs, in the sineGordon model. In this example, and others, the sineGordon model is usually obtained through the elimination of microscopic degrees of freedom. Although these degrees of freedom are irrelevant to equilibrium properties of the system, they influence the dynamical properties by acting as a heat bath and thus inducing dissipation. In the classical sine-Gordon model, it is known that dissipative effects play an important role in the dynamics. ${ }^{3}$ Therefore, it is important to investigate the effect of dissipation on the quantum dynamics of the system.

The influence of dissipation on quantum tunneling was studied by Caldeira and Leggett ${ }^{4}$ for systems with one degree of freedom. They found that it is convenient to include dissipation within the path-integral formulation of quantum mechanics. In the present work we extend the above formulation to the case of $1+1$ dimensions, and calculate the soliton pair creation rate under an external field in the semiclassical approximation.

We consider the one-dimensional (1D) quantum sineGordon model in an external field whose Lagrangian is given by

$\mathscr{L}=A \int_{-\infty}^{\infty} d x\left[\left(\frac{\partial \phi}{\partial t}\right)^{2}-\left(\frac{\partial \phi}{\partial x}\right)^{2}+2 \omega^{2}(: \cos \phi-1:)+\epsilon \phi\right]$,

where $\phi$ is the Bose field, $\epsilon$ the external field, $A$ a positive constant, and $\omega$ the frequency of small amplitude oscillations. The notation : : denotes the normal product, which is introduced to avoid the ultraviolet divergence. ${ }^{5}$ At $\epsilon=0$, the properties of this system have been studied by one of the authors and co-workers ${ }^{6}$ using the exact method. This method is, however, not suitable to investigate the behavior of the system in an external field. Therefore, we use the path-integral formulation in which quasiclassical results are easy to obtain, in close analogy to Ref. 2.

When $\epsilon$ is smaller than $\epsilon_{c}=2 \omega^{2}$, this system has an infinite series of metastable states with values of $\phi=\phi_{0}+2 n \pi$ ( $n$ :integer), where $\phi_{0}$ vanishes if $\epsilon=0$. The decay rate $P$ of one of those states, in the semiclassical approximation, is given by ${ }^{1}$

$$
P=K \exp \left(-S_{B}\right)
$$

In this equation, $K$ denotes the determinantal factor, multiplied by the size of the system, and $S_{B}$ the Euclidean action $S$, evaluated for a single "bounce."1,2,4 Specifically, $S$ is given by

$$
S=-\int_{-\infty}^{\infty} \mathscr{L}_{E} d \tau+S_{d},
$$

where $\mathscr{L}_{E}$ is obtained from $\mathscr{L}$ by $t \rightarrow-i \tau$, and the coupling to the heat bath is described by ${ }^{4}$

$$
S_{d}=\frac{\eta_{0}}{2 \pi} \iint_{-\infty}^{\infty} \int d \tau d \tau^{\prime} d x\left(\frac{\phi(x, \tau)-\phi\left(x, \tau^{\prime}\right)}{\tau-\tau^{\prime}}\right)^{2} .
$$

In the limit of small $\epsilon$, the bounce is a bubble with $\phi=2 \pi$ on the $x-\tau$ plane in a background with $\phi=0$, and the size of the bubble is much larger than its wall (thin-wall approximation). To be able to make contact with the limit of zero dissipation, we assume the wall to be of the form

$$
\phi=4 \tan ^{-1} \exp [r / 2 d(\theta)],
$$

where $r$ is the coordinate normal to the wall of the bubble; $r=0$ corresponds to the center of the wall and $\theta$ is the angle between the $x$ axis and the normal (Fig. 1). The thickness of the wall $d(\theta)$ will be determined later. However, evaluating the dissipative term with (5) is cumbersome, and we choose to evaluate $S_{d}$ by inserting $\phi=-r / d+\pi$ for $-\pi d<r<\pi d$. This approximation does not change the singularity in the weak-field limit which we are most interested in. Then the action is found to be given by

$$
\begin{aligned}
S / A= & \int_{0}^{\tau_{0}} 16\left(\frac{d^{-1}+4 \omega^{2} d}{\sin \theta}+\pi \eta h(y)\right)\left|x_{\tau}\right| d \tau \\
& -8 \pi \epsilon \int_{0}^{\tau_{0}} x d \tau .
\end{aligned}
$$

Here, $x=x(\tau)$ is the contour with $\phi=\pi, \tau_{0}$ the maximum value of $\tau$ on the contour [we assume $x\left(\tau_{0}\right)=0$ ], $x_{\tau}=d x$ / $d \tau=-\tan \theta, y=\tau \sin \theta / \pi d$, and $\eta=\eta_{0} / A$. It is assumed that the form of the bubble is symmetric with respect to the $x$ and $\tau$ axes. Thus, we could restrict ourselves in (6) (and in the following) to the region $x>0, \tau>0$. Finally, the function $h(y)$ behaves as lny for large arguments, and vanishes for $y \leq 1$, which reflects the fact that dissipation is ineffective as long as the wall is parallel to the $\tau$ axis. As 


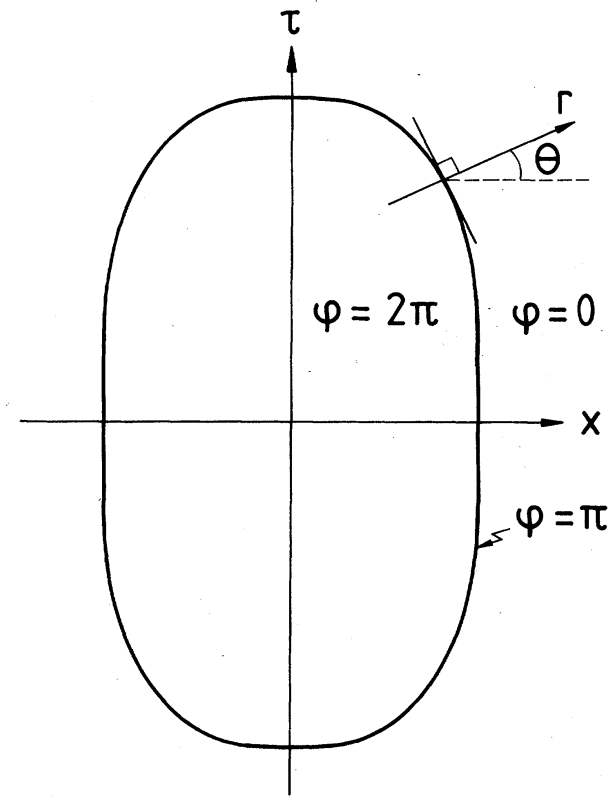

FIG. 1. Form of a bubble with $\phi=2 \pi$ in a background with $\phi=0$ on the $x-\tau$ plane. $r$ is the coordinate normal to the bubble surface. $\theta$ is defined as the angle between the $x$ and $r$ axes.

an approximate form, we take $h(y)=\Theta(y-1) \ln y$, where $\Theta()$ is the step function. By minimizing (6) with respect to $d(\theta)$, and as long as $y \geq 1$, we obtain

$$
d(\theta)=\frac{\pi \eta \sin \theta+\left(\pi^{2} \eta^{2} \sin ^{2} \theta+16 \omega^{2}\right)^{1 / 2}}{8 \omega^{2}} .
$$

For $\eta<<\omega$, as well as for $\theta=0$, the thickness of the wall is given by $d_{0}=(2 \omega)^{-1}$, as expected. On the other hand, we find for $\eta \gg \omega$ :

$$
d(\theta \approx \pi / 2) \approx(\eta / \omega) d_{0},
$$

in agreement with the results of Ref. 4. In the following, we determine the action of the bubble for weak and strong damping, $\eta<<\omega$ and $\eta>>\omega$, respectively.

(i) $\eta<<\omega$. In this limit, $d(\theta) \simeq d_{0}$, and we optimize the action with respect to the contour $x(\tau)$. We obtain the following differential equation:

$$
\begin{aligned}
64 \omega \frac{d}{d \tau} & \frac{x_{\tau}}{\left(1+x_{\tau}^{2}\right)^{1 / 2}}+8 \pi \epsilon \\
& -16 \pi \eta \frac{d}{d \tau}\left[\ln y+\left(1+x_{\tau}^{2}\right)^{-1}\right] \Theta(y-1)=0,
\end{aligned}
$$

with $y=2 \omega \tau\left|x_{\tau}\right| / \pi\left(1+x_{\tau}^{2}\right)^{1 / 2}$. Using this equation, we can write the action in the form

$$
S / A=64 \omega \int_{0}^{\tau_{0}}\left(1+x_{\tau}^{2}\right)^{-1 / 2} d \tau
$$

Equation (9) can be integrated once. For simplicity, we neglect the second term in the square bracket of (9), and obtain for small $\tau, \tau<\tau_{c}=2 / \epsilon^{1 / 2}$ :

$$
\frac{x_{\tau}}{\left(1+x_{\tau}^{2}\right)^{1 / 2}}=-\frac{\pi \epsilon}{8 \omega} \tau \text {, }
$$

while for large $\tau, \tau>\tau_{c}$, the dissipation has to be taken into account. Then we obtain

$$
\frac{x_{\tau}}{\left(1+x_{\tau}^{2}\right)^{1 / 2}}-\frac{\pi \eta}{4 \omega} \ln y=-\frac{\pi \epsilon}{8 \omega} \tau
$$

In the limit of zero dissipation, we find from $x_{\tau}(\tau$ $\left.=\tau_{0}\right)=-\infty$ that $\tau_{0}=8 \omega / \pi \epsilon$, and also reproduce the result of Maki, ${ }^{2}$ in our notation given by $S_{B} / A=128 \omega^{2} / \epsilon$. However, for finite $\eta$, this is only valid as long as

$$
\frac{\pi \eta}{4 \omega} \ln \left(\frac{16 \omega^{2}}{\pi^{2} \epsilon}\right) \leq 1
$$

and it is clear that this condition cannot be satisfied for very small $\epsilon$. To investigate this limit, we neglect the first term in (12), and find that $\tau_{0}$ is determined by the equation

$$
\tau_{0}=\frac{2 \eta}{\epsilon} \ln \left(\frac{2 \omega}{\pi} \tau_{0}\right),
$$

which is easily iterated. Finally, the action can be calculated, and we obtain the following result:

$$
S_{B} / A=\frac{128 \omega \eta}{\epsilon} \ln \left(\frac{4 \omega \eta}{\pi \epsilon}\right) .
$$

We remark that this expression is valid provided $\epsilon \leqslant \epsilon_{c}(\eta / \omega) \exp (-4 \omega / \pi \eta)$, where $\epsilon_{c}$ is the classical depinning field as introduced above. Taking only the exponential factor in (2) into account, we obtain for the decay rate

$$
P \sim\left(\frac{4 \eta \omega}{\pi \epsilon}\right)^{-128 \eta \omega A / \epsilon}
$$

Note, however, that the determinantal factor in $K$ depends weakly on the external field. For $\eta=0$, the result is ${ }^{2} K \sim \epsilon$.

(ii) $\eta \gg \omega$. In this limit, the contribution of the first three terms in (6) is very large $(\sim \eta /$ length) except in the case where the wall is parallel to the $\tau$ axis $(\sim \omega /$ length).

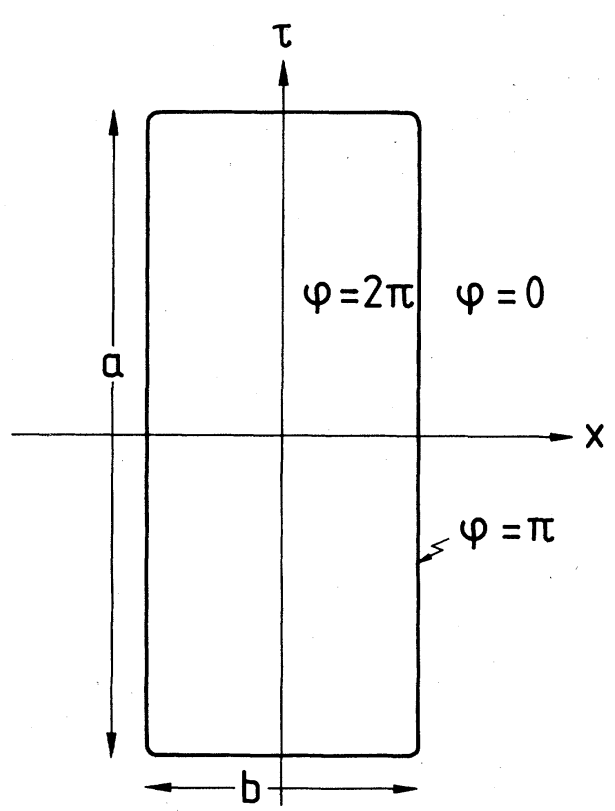

FIG. 2. Form of the bubble in the strongly damped limit $(\omega<<\eta)$. 
TABLE I. Correspondence between the commensurability pinned CDW and the present system. $N$ : commensurability; $c_{0}$ : phason velocity; $e$. electronic charge; $E$ : electric field; $v_{F}$ : Fermi velocity; $\tau_{2}:$ backward scattering time due to impurities.

\begin{tabular}{|c|c|c|c|c|}
\hline $\begin{array}{l}\text { Commensurability } \\
\text { pinned CDW }\end{array}$ & $N \phi$ & $\frac{v_{F}}{4 \pi N^{2} c_{0}}$ & $\frac{4 e N c_{0}^{2}}{v_{F}} E$ & $\frac{c_{0}^{2}}{\tau_{2} v_{F}^{2}}$ \\
\hline Present system & $\phi$ & $A$ & $\epsilon$ & $\eta$ \\
\hline
\end{tabular}

Thus, we can minimize the action by maximizing the length of the wall which is parallel to the $\tau$ axis. As a result, a rectangular bubble elongated along the $\tau$ axis is the most suitable one (Fig. 2).

The action can be estimated to give

$S(a, b) / A=8 \pi b \eta+32 \omega a+8 \pi b \eta \ln \left(2 \omega^{2} a / \pi^{2} \eta\right)-2 \pi \epsilon a b$

where $a$ and $b$ are the lengths of the bubble along the $\tau$ and $x$ axes, respectively. It is straightforward to minimize this expression with respect to $a$ and $b$, and we find the following result:

$$
\begin{aligned}
& a \simeq \frac{4 \eta}{\epsilon} \ln \left(\frac{2 e \omega^{2}}{\pi^{2} \eta} a\right), \\
& b \simeq \frac{16 \omega}{\pi \epsilon},
\end{aligned}
$$

which shows the expected behavior, namely, $a \gg b$. The value of the action at the minimum, and the decay rate are given by

$$
S_{B} / A=\frac{128 \eta \omega}{\epsilon} \ln \left(\frac{8 e \omega^{2}}{\pi^{2} \epsilon}\right)
$$

and

$$
P \sim\left(\frac{8 e \omega^{2}}{\pi^{2} \epsilon}\right)^{-128 \eta \omega A / \epsilon}
$$

These expressions are essentially the same as those for the weak-field limit for $\eta<<\omega$, since we have neglected terms of the order $\epsilon^{-1}$ in the action. Note that, as in the case of one degree of freedom, ${ }^{4}$ damping strongly reduces the decay rate, as can be seen from (19) which, up to numerical factors, can be expressed as

$$
S_{B}(\eta>>\omega) \sim S_{B}(\eta=0) \frac{\eta}{\omega} \ln \left(\frac{\omega^{2}}{\epsilon}\right) \text {. }
$$

This should be compared with the result of Ref. 4, which also shows that the action is increased by a factor of order $\eta / \omega$, in the strong dissipation limit.

In order to discuss the experimental implication of our results, we show the correspondence between our Lagrangian and that of the commensurability pinned charge-density wave in Table $\mathrm{I}^{2,7-10}$ In the latter system, $P$ is proportional to the electric conductivity except a prefactor which is weakly dependent on $\epsilon$.

In the absence of dissipation, the typical scale of the electric field is given by $\epsilon_{1}=128 \omega^{2} A$, which is for small $N$ larger than the classical depinning field $\epsilon_{c}$ :

$$
\frac{\epsilon_{1}}{\epsilon_{c}}=64 A=\frac{16 v_{F}}{\pi c_{0} N^{2}}
$$

Although $v_{F} \simeq 10 \ldots 10^{2} c_{0}$, quantum tunneling phenomena could be observed before the CDW is depinned classically if commensurability is high enough. On the other hand, our results show that the typical scale of electric field in the dissipative case is given by $\epsilon_{d}=128 \eta \omega A$, which is smaller than $\epsilon_{1}$ for small $\eta$.

Unfortunately, in systems in which quantum tunneling is large enough to be observed by usual experiments, the quantumness is so large that the semiclassical approximation becomes doubtful. Thus, the construction of the fully quantum-mechanical approach is desirable. We may expect, however, that the functional dependence of $P$ on $\epsilon$ would be unchanged if parameters are renormalized by the quantum fluctuation because these renormalizations do not depend on the external field as far as the external field is small enough.

We thank A. Schmid and W. Wonneberger for useful discussions. One of us (K.H.) is grateful to the Alexander von Humboldt Foundation for financial support.
"Permanent address: Department of Engineering Science, Faculty of Engineering, Hokkaido University, Sapporo 060, Japan.

${ }^{1}$ S. Coleman, in The Whys of Subnucler Physics, edited by A. Zichichi (Plenum, New York, 1979), and references therein.

${ }^{2}$ K. Maki, Phys. Rev. B 18, 1641 (1978).

${ }^{3}$ M. Imada, J. Phys. Soc. Jpn. 50, 401 (1981); see also M. Büttiker and R. Landauer, Phys. Rev. A 23, 1397 (1981).

${ }^{4}$ A. O. Caldeira and A. J. Leggett, Phys. Rev. Lett. 46, 211 (1981); Ann. Phys. (N.Y.) 149, 374 (1983).

${ }^{5}$ R. F. Dashen, B. Hasslacher, and A. Neveu, Phys. Rev. D 11, 3424 (1975).

${ }^{6}$ M. Imada, K. Hida, and M. Ishikawa, J. Phys. C 16, 35 (1983);
K. Hida, M. Imada, and M. Ishikawa, ibid. 16, 4945 (1983); see also M. Fowler and X: Zotos, Phys. Rev. B 25, 5806 (1982).

${ }^{7}$ P. A. Lee, T. M. Rice, and P. W. Anderson, Solid State Commun. 14, 703 (1974).

${ }^{8}$ H. Fukuyama, J. Phys. Soc. Jpn. 41, 513 (1976).

${ }^{9} \mathrm{U}$. Eckern and A. Geier, in Proceedings of the 17th International Conference on Low Temperature Physics, Karlsruhe, August 1984, edited by U. Eckern, A. Schmid, W. Weber, and H. Wühle (North-Holland, Amsterdam, 1984), p. 1343.

${ }^{10}$ In the context of CDW systems, the concept of collective Zener tunneling has been employed by J. Bardeen, Phys. Rev. Lett. 45, 1978 (1980); see also W. Wonneberger, Z. Phys. B 50, 23 (1983). 